\title{
Viral load-guided immunosuppression after lung transplantation (VIGILung)—study protocol for a randomized controlled trial
}

\author{
Jens Gottlieb ${ }^{1,2^{*}}$ (D), Alexander Reuss ${ }^{3}$, Konstantin Mayer $^{4}$, Karin Weide ${ }^{3}$, Carmen Schade-Brittinger ${ }^{3}$, \\ Susanne Hoyer ${ }^{1,2}$ and Peter Jaksch ${ }^{5}$
}

\begin{abstract}
Background: Immunosuppression including high-dose calcineurin inhibitors (CNI) is essential after lung transplantation. Dosing is usually guided by therapeutic drug monitoring adjusted to target trough levels of CNIs to keep the balance between over-dose causing severe toxicity and increased risk of infections or under-dose with a risk of graft injury. Adaptation of CNI-based immunosuppression by monitoring of torque teno virus (TTV), a latent nonpathogenic DNA virus, measured in the whole blood in addition to conventional therapeutic drug monitoring may reduce the toxicity of immunosuppression with similar efficacy.
\end{abstract}

Methods/design: An open-label, randomized, controlled, parallel-group, multicenter trial in lung transplant recipients will be conducted to investigate the safety and efficacy of immunosuppression guided by TTV monitoring as an add-on to conventional therapeutic drug monitoring. Adult lung transplant recipients 21 to 42 days after transplantation are eligible to participate. Patients $(N=144)$ will be randomized 1:1 to the experimental intervention (arm 1: immunosuppression guided by TTV monitoring in addition to conventional therapeutic drug monitoring of tacrolimus trough levels) and control intervention (arm 2: conventional therapeutic drug monitoring). Outcomes will be assessed 12 months after randomization with the change in glomerular filtration rate as the primary endpoint. Secondary endpoints will be additional measurements of renal function, allograft function, incidence of acute rejections, incidence of chronic lung allograft dysfunction, graft loss, and infections.

Discussion: The results of this randomized controlled trial may reduce the toxicity of immunosuppression after lung transplantation while maintaining the efficacy of immunosuppression. Study results are transferable to all other solid organ transplantations.

Trial registration: ClinicalTrials.gov NCT04198506. Registered on 12 December 2019

Keywords: Lung transplantation, Immunosuppression, Graft rejection, Kidney failure, Torque teno virus, Randomized controlled trial

\footnotetext{
* Correspondence: gottlieb.jens@mh-hannover.de

'Department of Respiratory Medicine OE6870, Hannover Medical School,

30625 Hannover, Germany

${ }^{2}$ BREATH (Biomedical Research in End-stage and obstructive Lung Disease

Hannover), Hannover, Germany

Full list of author information is available at the end of the article
}

(c) The Author(s). 2021 Open Access This article is licensed under a Creative Commons Attribution 4.0 International License, which permits use, sharing, adaptation, distribution and reproduction in any medium or format, as long as you give appropriate credit to the original author(s) and the source, provide a link to the Creative Commons licence, and indicate if changes were made. The images or other third party material in this article are included in the article's Creative Commons licence, unless indicated otherwise in a credit line to the material. If material is not included in the article's Creative Commons licence and your intended use is not permitted by statutory regulation or exceeds the permitted use, you will need to obtain permission directly from the copyright holder. To view a copy of this licence, visit http://creativecommons.org/licenses/by/4.0/ The Creative Commons Public Domain Dedication waiver (http://creativecommons.org/publicdomain/zero/1.0/) applies to the data made available in this article, unless otherwise stated in a credit line to the data. 


\section{Background}

High-dose immunosuppression containing calcineurin inhibitors (CNI) is essential after lung transplantation. Dosing is usually guided by fixed target levels, established to keep the balance between over-dose causing toxicity and increased risk of infections or under-dose with a risk of graft injury.

Therapeutic drug monitoring represents the current gold standard of guiding immunosuppression after solid organ transplantation [1]. Despite rigorous monitoring, acute rejection occurs in approximately one third of patients in the first year after lung transplantation [2]. Immunosuppressive regimens are responsible for considerable toxicity. Approximately $24 \%$ of the recipients will develop kidney failure within 1 year of transplantation and $2 \%$ end-stage kidney disease. Increased infection rates are associated with over-immunosuppression, and infections are the leading cause of death during the first postoperative year.

Clinical experience suggests that individual tailoring of immunosuppression could potentially optimize patient outcome [3-6]. Reliable, reproducible, cost-effective, and non-invasive biomarkers are needed to reduce the risk of graft injury and toxicity to guide immunosuppression.

Latent DNA viruses in the whole blood (e.g., torque teno virus (TTV)) are detectable in the vast majority of lung transplant recipients [3].The load of these latent viruses was associated with the strength of immunosuppression in solid organ recipients [7].

DNA viruses in the whole blood (torque teno virus/ TTV, HHV-6, EBV) and urine (BK virus) can be detected in the majority of humans including transplant recipients. Some of them do not cause symptoms of infection, for example, TTV. The viral load of TTV was used as a surrogate biomarker of cell-mediated immunity (load increasing with the strength of immunosuppression) but has never been studied in a prospective trial. It is suggested by pivotal studies [3] that CNI-based immunosuppression may be reduced in the majority of lung transplant recipients during the first postoperative year.

Guiding immunosuppression by an immune response assay in a prospective trial resulted in reductions in CNI doses of $13-25 \%$ within the first year after liver transplantation, with documented reductions in bacterial and fungal infections [6]. In lung transplant recipients, a CNI reduction of $50 \%$ has led to an improvement of GFR (CKD-EPI) by $10 \mathrm{ml} / \mathrm{min} / 1.73 \mathrm{~m}^{2}$ after 12 months in a recent randomized trial [8]. With the management of trough levels by TTV load, it is expected that a similar CNI dose reduction can be achieved.

The results of the VIGILung trial may have an impact on therapeutic strategies for patients after lung transplantation. Study results may be transferable to all other solid organ transplantations.

\section{Methods/design Objectives}

The aim of this randomized controlled trial is the prospective investigation of the safety and efficacy of an individual adaptation of the tacrolimus-based immunosuppression by a non-invasive biomarker (torque teno virus (TTV) load in the whole blood).

The primary endpoint will be $\Delta$-glomerular filtration rate (GFR) defined as the change of the glomerular filtration rate GFR between randomization and 12 months thereafter as an indicator for toxicity. GFR will be estimated using the Chronic Kidney Disease Epidemiology Collaboration (CKD-EPI) formula [9].

Secondary outcomes include further parameters on renal function, parameters regarding lung allograft function forced expiratory volume in $1 \mathrm{~s}$ (FEV1) in \% of baseline, incidence of acute rejections, lymphocytic bronchiolitis and/ or chronic lung allograft dysfunction, re-transplantation or death due to graft failure, infections (cytomegalovirus (CMV), community-acquired respiratory viruses (CARV), fungal or bacterial infections), incidence of unscheduled emergency hospitalizations or admissions for intensive care unit (ICU), Quality of Life Questionnaire (EQ-5D) and exercise capacity, the state of immune-system (cluster of differentiation 4 (CD4)-lymphocytes, donor-specific antibodies (DSA), IgG level and use of rescue immune therapy), and details on the tacrolimus immunosuppression (tacrolimus trough levels, tacrolimus doses, and number of adjustment of immunosuppression).

\section{Design and participants}

The screening of the patients will be performed in two participating study sites (Hannover Medical School and University of Vienna). We assume that screening of 250 patients will result in 144 subjects eligible for the study. The recruitment period is expected to be 39 months.

Selected in- and exclusion criteria are as follows:

Inclusion criteria:

- Adult patients 21 to 42 days after de novo lung transplantation (bilateral or combined)

- Tacrolimus based immunosuppression

- Detectable TTV load at randomization (> $2.7 \log 10$ copies/mL)

- Women of child-bearing potential: negative serum pregnancy test and highly effective methods of contraception throughout the study.

\section{Exclusion criteria:}

- History or high-risk of obstructive airway complications after lung transplantation 
- Respiratory failure (need for oxygen therapy or ventilation at screening) inability to undergo transbronchial biopsy

- Advanced kidney failure (GFR CKD-EPI $<30 \mathrm{ml} /$ $\mathrm{min} / 1.73 \mathrm{~m}^{2}$ at inclusion and/or current renal replacement therapy at inclusion or randomization

- Advanced liver cirrhosis (CHILD-Pugh Score C) after lung transplantation

- Fluctuating tacrolimus drug levels (less than $20 \%$ in target range after transplantation)

- Symptoms of significant mental illness with the inability to cooperate or communicate with the investigator

- Unlikeliness to comply with the study requirements

- HIV positivity

\section{Randomization}

Randomization will be performed centrally via telefax by the Center for Clinical Trials (KKS) of the PhilippsUniversity Marburg for patients matching all eligibility criteria and will be stratified by high-risk CMV status $(\mathrm{D}+\mathrm{R}-)$ (yes/no) and center using randomization lists generated at KKS with permuted blocks of randomly varying size. The chance for allocation to the control and experimental group is 1:1. Investigators at the participating sites request the randomization result by faxing the completed randomization form, whereupon KKS staff will consult the randomization database and transmit the result to the investigator.

\section{Trial intervention and control}

After lung transplantation (day 0), all patients will receive a standard tacrolimus-based triple immunosuppressive regime (tacrolimus, prednisolone, and a cell cycle inhibitor (depending on induction therapy with alemtuzumab)). Tacrolimus-based immunosuppressive protocols represent the standard of care worldwide [2] and were therefore chosen as the comparator. Inclusion and screening will occur 21 to 42 days after lung transplantation; a further screening visit will occur 4 weeks later, especially to evaluate the achievement of a sufficient TTV level.

\section{Follow-up protocol}

At visit 3 patients will be assigned to one of the two treatment groups detailed in Fig. 1.

Study visits will be performed at months 1,2 , and 3 after transplantation and at months 3, 6, 9, and 12 after randomization. The final examination will be performed at month 12 after randomization or in case of a premature end of study treatment.

In both treatment arms, during maintenance, immunosuppression interruption of one out of three immunosuppressive components for up to 4 weeks will be allowed, e.g., in case of cell cycle inhibitors induce severe toxicity (e.g., leukopenia $<4000 / \mu \mathrm{l}$, thrombocytopenia < $50,000 / \mu \mathrm{l}$, anemia with hemoglobin $<8 \mathrm{~g} / \mathrm{dl}$ ).

After transplantation, tacrolimus and additional immunosuppressive drugs, usually a cell cycle inhibitor (mycophenolate mofetil, mycophenolate sodium, azathioprine) and prednisolone, are given.

In case of induction therapy with alemtuzumab, tacrolimus and prednisolone are given from day 0 and cell cycle inhibitors are delayed until the restoration of lymphocyte count-usually after 12 months $[5,10]$.

Adjustment of tacrolimus immunosuppression will be established in predefined steps, derived from previous
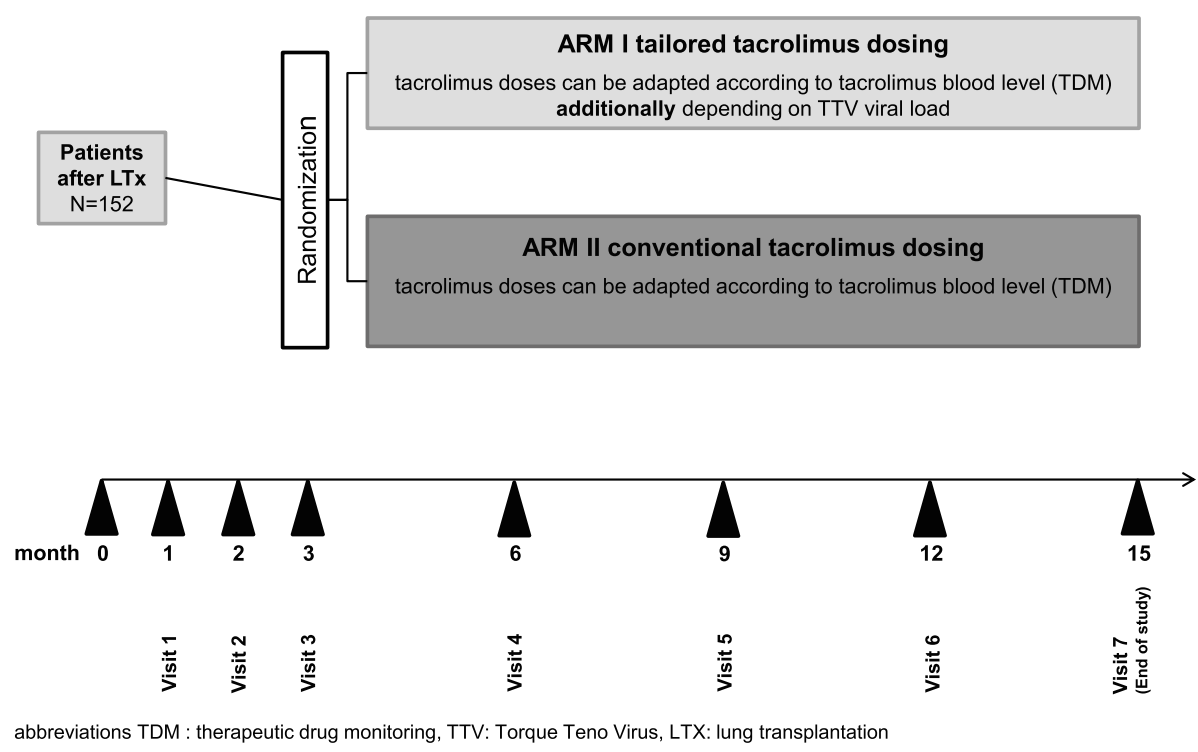

Fig. 1 Trial flow chart and treatment groups 
trials in immunosuppression [11]. Immunosuppression (IS) will be managed according to predefined steps (with gradually reduced tacrolimus target levels from step 8 to 1. Patients without induction with alemtuzumab will start on step 6 (target trough level of tacrolimus 8-12 $\mathrm{ng} / \mathrm{ml}$ ), and patients with induction with alemtuzumab will start on step 4 (target trough level of tacrolimus 6$10 \mathrm{ng} / \mathrm{ml}$ ) in this scheme. Trough levels of tacrolimus will be determined according to liquid chromatography coupled with mass spectrometry (LC-MS) at least once a month.

All patients who experience an acute cellular rejection after reduction of CNI will be put on increased doses of calcineurin inhibitors.

In arm II, the tacrolimus-based immunosuppression will be guided by conventional therapeutic drug monitoring (TDM). In patients randomized to the conventional CNI dosing, the immunosuppressive drugs should be applied according to trough levels, signs of toxicity, and center practice.

In arm I, the immunosuppression will be guided by the torque teno virus (TTV) monitoring and conventional therapeutic drug monitoring (TDM) (Fig. 2). Reduction in target trough levels will only be performed in stable patients after exclusion of silent rejection in the transbronchial biopsy.

The TTV load reached after TTV stabilization (expected after 3 months) will be used to guide immunosuppression, which will be tailored according to the scheme above (Fig. 2).

Maintenance immunosuppression will be guided by therapeutic drug monitoring (TDM) of tacrolimus trough levels. Furthermore, signs of toxicity of tacrolimus and cell cycle inhibitors (e.g., leukopenia, gastrointestinal side effects, hypogammaglobulinemia) may lead to dose adaptation and switch between various cell cycle inhibitors.

\section{Measurements}

Most important measurement is creatinine (from blood chemistry) as the key parameter to determine the primary endpoint $\Delta$ GFR between randomization and 12 months thereafter.

Another key measurement within this trial is the TTV load in arm 1 patients. TTV load is classified as low with TTV load $<7 \log _{10}$, as medium with TTV load from 7 to $9.5 \log _{10}$, and as high with a TTV load of $>9.5 \log _{10}$.

TTV DNA quantitation will be done by TaqMan realtime PCR using probe and primers as described previously [12]. The linear range of TTV quantitation ranges from 2.7 to $10.7 \log 10$ copies $/ \mathrm{mL}$ as determined by the use of 10-fold dilutions of a plasmid standard. The limit of detection in plasma is $2.7 \log 10$ copies $/ \mathrm{ml}$. In each run, a TTV DNA standard and positive and negative controls will be included and any signs of PCR inhibition will be assessed by quantitation of the known amount of control DNA spiked into the samples before DNA extraction [12].

\section{Outcome assessments}

For the primary endpoint $\Delta$ GFR between randomization and 12 months thereafter, creatinine from the serum will be measured to calculate GFR (CKD-EPI) at each visit.

The main secondary outcomes are assessed as follows:

- GFR (CKD-EPI) at 1 and 2 months after transplantation (screening visits) and 0, 3, 6, 9, and 12 months after randomization

- GFR (cystatin) at randomization and 12 months after randomization

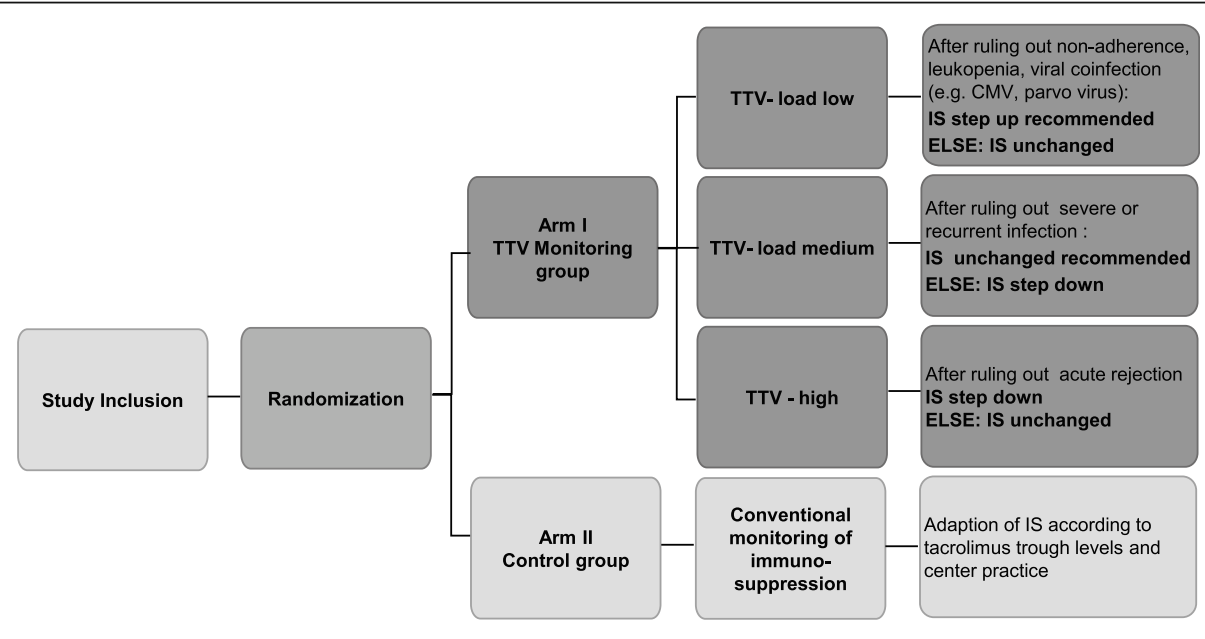

abbreviations IS: immunosuppression, TTV: Torque Teno Virus, CMV: cytomegalovirus

Fig. 2 Adaptation of immunosuppression in the treatment groups 
- Proportion of patients with biopsy-proven acute cellular rejection (grade A1 or higher) within 12 months after randomization

- Proportion of patients with an episode of biopsyproven lymphocytic bronchitis (grade B1R or higher) within 12 months after randomization

- Proportion of patients with cytomegalovirus (CMV) infection and number of CMV disease episodes within 12 months after randomization

- Proportion of patients with community-acquired respiratory viral infection (CARV) within 12 months after randomization

- Proportion of patients with fungal and bacterial infections within 12 months after randomization

- Proportion of patients with any of the abovementioned infections within 12 months after randomization

- Proportion of patients with unscheduled or emergency hospitalizations after randomization

- Proportion of patients with ICU admissions after randomization

- Quality of life (EQ-5D visual analog scale) at screening visits and 0, 3, 6, 9, and 12 months after randomization

- Proportion of patients with new or progressive malignancy within 12 months after randomization

- Tacrolimus trough levels at screening visits and 0,3, 6, 9, and 12 months after randomization

- Daily tacrolimus dose [mg] at screening visits and 0 , 3, 6, 9, and 12 months after randomization

- Proportion patients with increased/unchanged/ decreased (compared to the previous visit) target trough levels of tacrolimus at screening visits and 0 , 3, 6, 9, and 12 months after randomization

- Exercise capacity measured by the percent predicted distance achieved in the 6-min walk test at randomization and 12 months thereafter

- CD4 lymphocyte count at 0, 6, and 12 months after randomization

- Proportion of patients with presence of donor-specific antibodies at 0,6 , and 12 months after randomization

- FEV1 in \% baseline value at screening visits and 0, 3, 6, 9, and 12 months after randomization

- Incidence of chronic lung allograft dysfunction between randomization and 12 months thereafter

- IgG level at 0, 6, and 12 months after randomization

- Proportion of patients with rescue immunotherapy (defined by the use of ATG, rituximab, alemtuzumab, plasma exchange, immunoadsorption) between randomization and 12 months thereafter

Data collection time points are displayed in Fig. 3.

\section{Statistical analysis}

Unblinding procedures are not applicable because the trial is not blinded. Data analysts will be blinded for the randomization results until database closure for the final analysis. Apart from safety reports to the Data and Safety Monitoring Committee (DSMC), no interim analyses are planned.

\section{Primary efficacy analysis}

The primary efficacy endpoint $\Delta$ GFR is defined as the change of the glomerular filtration rate GFR between randomization and 12 months thereafter. GFR will be estimated using the CKD-EPI formula. The primary endpoint analysis will be adjusted for randomization strata and baseline. No subgroup analyses are planned.

An analysis of covariance (ANCOVA) with strata used at randomization and treatment as fixed factors, and the baseline GFR as a covariate is used to test for a difference in location of $\Delta$ GFR (in $\mathrm{ml} / \mathrm{min} / 1.73 \mathrm{~m}^{2}$ ) between the groups (two-sided at a 0.05 significance level). The analysis of the primary endpoint will be based on the intention-to-treat population.

\section{Secondary endpoints}

Categorical variables will be reported as absolute and relative frequency, continuous variables by median, mean, standard deviation, 95\% confidence interval, minimum, and maximum. Student's $t$ test, analysis of covariance or their non-parametric analogs, as well as chisquare or Fisher's exact test, furthermore (generalized) linear mixed models will be applied as appropriate for the types of variables, group, high-risk CMV status, and time, as well as a group-by-time interaction term will be used as covariates in the mixed models. All statistical tests will be carried out at a two-sided significance level of $5 \%$ without adjustment for multiple comparisons. Full details will be stated in a statistical analysis plan.

\section{Safety analyses}

The as-treated population is defined as all included patients who have undergone at least one blood sample for therapy monitoring after randomization. Analysis of safety will be based on the as-treated population, i.e., patients will be analyzed according to the intervention they actually received.

\section{Sample size calculation}

The sample size calculation is performed assuming a $t$ test of $\Delta$ GFR. Because adjusting for stratification factors is assumed to reduce variability, the power for the ANCOVA test of the treatment variable is expected to be rather higher than lower compared to the $t$ test.

The sample size calculation is based on the assumption of a mean decline in GFR of 15 between randomization and 12 months thereafter and a standard deviation of 20 in the control group. Two randomized trials $[8,13]$ observed effect sizes of a similar magnitude. 


\begin{tabular}{|c|c|c|c|c|c|c|c|}
\hline \multirow[b]{3}{*}{ Time point } & \multicolumn{7}{|c|}{ STUDY PERIOD } \\
\hline & \multicolumn{2}{|c|}{ Enrolment } & \multirow{2}{*}{\begin{tabular}{|l|}
\multicolumn{1}{|c|}{$\begin{array}{c}\text { Allo- } \\
\text { cation }\end{array}$} \\
Visit 3 \\
Randomization \\
POD 90 \\
+/- 10 days
\end{tabular}} & \multicolumn{3}{|c|}{ Post-allocation } & \multirow{2}{*}{\begin{tabular}{|l|} 
Close-out \\
Visit 7 \\
End of study or \\
premature end \\
POD 450 \\
$+/-10$ days \\
\end{tabular}} \\
\hline & $\begin{array}{l}\text { Visit } 1 \\
\text { Screening I } \\
\text { POD } 32 \\
\text { +/- } 10 \text { days }\end{array}$ & $\begin{array}{l}\text { Visit 2 } \\
\text { Screening II } \\
\text { POD } 60 \\
+ \text { +- } 10 \text { days }\end{array}$ & & \begin{tabular}{|l} 
Visit 4 \\
POD 180 \\
$+/-10$ days
\end{tabular} & $\begin{array}{l}\text { Visit } 5 \\
\text { POD 270 } \\
+/-10 \text { days }\end{array}$ & $\begin{array}{l}\text { Visit } 6 \\
\text { POD } 360 \\
+/-10 \text { days }\end{array}$ & \\
\hline \multicolumn{8}{|l|}{ ENROLMENT: } \\
\hline \begin{tabular}{|l|} 
Patient information \\
and consent
\end{tabular} & $\mathbf{x}$ & & & & & & \\
\hline $\begin{array}{l}\text { Inclusion and } \\
\text { exclusion criteria }\end{array}$ & $\mathbf{x}$ & & & & & & \\
\hline Enrolment & $\mathbf{x}$ & & & & & & \\
\hline $\begin{array}{l}\text { Allocation } \\
\text { (Randomization) }\end{array}$ & & & $\mathbf{x}$ & & & & \\
\hline \\
\hline $\begin{array}{l}\text { TTV-Load guided } \\
\text { Immunosuppres- } \\
\text { sion (Arm 1) }\end{array}$ & & & & & & & \\
\hline \multicolumn{8}{|l|}{$\begin{array}{l}\text { Therapeutic drug } \\
\text { Monitoring }\end{array}$} \\
\hline $\begin{array}{l}\text { Monitoring } \\
(\text { Arm 1+ 2) }\end{array}$ & & & & & & & \\
\hline \multicolumn{8}{|l|}{ ASSESSMENTS } \\
\hline GFR (CDK EPI) & $\mathbf{x}$ & $\mathbf{x}$ & $\mathbf{x}$ & $\mathbf{x}$ & $\mathbf{x}$ & $\mathbf{x}$ & $\mathbf{x}$ \\
\hline \begin{tabular}{|l|} 
Bronchoscopy with \\
Transbronchial \\
Biopsy and Lavage
\end{tabular} & $\mathbf{x}$ & & $\mathbf{x}$ & $\mathbf{x}$ & & $\mathbf{x}$ & \\
\hline $\begin{array}{l}\text { General medical } \\
\text { history, GFR pre-Tx } \\
\text { and transplant } \\
\text { information } \\
\end{array}$ & $\mathbf{x}$ & & & & & & \\
\hline $\begin{array}{l}\text { Total Lung } \\
\text { Capacity }\end{array}$ & $\mathbf{x}$ & & $\mathbf{x}$ & & & & $\mathbf{x}$ \\
\hline FVC, FEV1 & $\mathbf{x}$ & $\mathbf{x}$ & $\mathbf{x}$ & $\mathbf{x}$ & $\mathbf{x}$ & $\mathbf{x}$ & $\mathbf{x}$ \\
\hline $\begin{array}{l}\text { Phys. Examination, } \\
\text { blood chemistry \& } \\
\text { blood count }\end{array}$ & $\mathbf{x}$ & $\mathbf{x}$ & $\mathbf{x}$ & $\mathbf{x}$ & $\mathbf{x}$ & $\mathbf{x}$ & $\mathbf{x}$ \\
\hline IgG,CD4 count & $\mathbf{x}$ & & $\mathbf{x}$ & & $\mathbf{x}$ & & $\mathbf{x}$ \\
\hline $\begin{array}{l}\text { HLA antibody } \\
\text { screen }\end{array}$ & $\mathbf{x}$ & & $\mathbf{x}$ & & $\mathbf{x}$ & & $\mathbf{x}$ \\
\hline $\begin{array}{l}\text { Tacrolimus trough } \\
\text { level }\end{array}$ & $\mathbf{x}$ & $\mathbf{x}$ & $\mathbf{x}$ & $\mathbf{x}$ & $\mathbf{x}$ & $\mathbf{x}$ & $\mathbf{x}$ \\
\hline TTV viral load & $\mathrm{x}^{1}$ & $\mathbf{x}^{1}$ & $x^{1}$ & $\mathrm{x}^{2}$ & $x^{2}$ & $x^{2}$ & $x^{2}$ \\
\hline EQ-5D & $\mathbf{x}$ & $\mathbf{x}$ & $\mathbf{x}$ & $\mathbf{x}$ & $\mathbf{x}$ & $\mathbf{x}$ & $\mathbf{x}$ \\
\hline $\begin{array}{l}\text { Adverse Events } \\
\text { (CTCAE 5.0) } \\
\end{array}$ & $\mathbf{x}$ & $\mathbf{x}$ & $\mathbf{x}$ & $\mathbf{x}$ & $\mathbf{x}$ & $\mathbf{x}$ & $\mathbf{x}$ \\
\hline 6-Minute Walk Test & $\mathbf{x}$ & 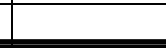 & $\mathbf{x}$ & & & & $x$ \\
\hline
\end{tabular}

Fig. 3 Data collection time points (SPIRIT figure) 
Compared to the control group, a mean decline in GFR of 5 in the experimental group (i.e., difference of 10 in GFR declines between the intervention groups) is regarded as clinically relevant. For a 2 -sided significance level of $5 \%$ and a power of $80 \%, 64$ patients per group $(n=128$ in total) are needed to achieve a power of $80 \%$ if the true difference in means is 10 and the standard deviation is 20 in both. With an estimated dropout rate of $10 \%, 144$ patients have to be randomized.

\section{Data collection}

Patient data are collected during the planned study visits on site using electronic case report forms (e-CRF) hosted by KKS Marburg. The data are entered directly at the participating sites via a web browser to the e-CRF and are transferred via TLS encryption to the central database.

Access to the e-CRF is only allowed for documented trial personnel using individual user accounts.

In a multistage procedure, the given data will be checked electronically for their plausibility and consistency. Even during data collection, implausible data will be flagged automatically by implemented validation checks. In the next stage, detected inconsistencies and missing or implausible data will be clarified with queries and necessary changes will be carried out.

In order to ensure the anonymity of the patient data, the patient data in the e-CRF are recorded with a patient number consisting of a center number and a consecutive number. An allocation list (Rando-Log) containing the patient number and the identifying data of the patient is only kept in the participating site.

Missing data will be addressed by intention-to-treat (ITT) analysis by single and multiple imputations, weighted estimating equations or model-based strategies. Specific details on the handling of missing data will be given in a statistical analysis plan that will be finalized prior to database lock.

\section{Safety endpoints}

Safety data will be summarized by using descriptive statistical methods. All adverse events (AEs) occurring during the conduct of the clinical trial will be monitored carefully and recorded on electronic case report forms (eCRFs). The causality assessment is performed by the investigator of the trial site concerned. In their clinical routine, the participating sites work with standardized questionnaires to systematically elicit information about potential adverse events. All adverse events (AE) are collected in the e-CRF and marked if serious or not. AEs which are frequent and of clinical interest (e.g., hematological and renal toxicities) will be reported in the final publication of the trial results.
All serious adverse events (SAEs) will be reported by the investigators to the Philipps-University of Marburg (KKS Marburg) within 24 h of becoming known. SAEs are recorded in the study-specific safety database. The SAE assessment is performed by KKS Marburg. The collection and handling of serious adverse events (SAE) follow procedures described in the study-specific SAE manual. SAEs are collected in a pharmacovigilance software which is validated for reporting obligations to the authorities.

All suspected adverse reactions related to an investigational medicinal product that are both unexpected and serious (SUSARs) will be notified by KKS Marburg to the competent authority, the ethics committee, and all investigators involved.

The study conduct and safety of participants will be monitored by a Data and Safety Monitoring Committee (DSMC). The DSMC consists of two clinical experts and one statistician independent from the coordinating investigator supervising the conduct of the study. Furthermore, clinical monitoring of patient data will be performed by on-site or remote by sponsor staff. Sponsor staff specializing in quality management will audit trial sites' adherence to planned processes on site.

The DSMC will meet via video conference on a regular basis (first meeting 6 months after inclusion of the first patient and once per year after that) and will be responsible for the independent evaluation of the safety of patients taking part in the clinical trial. The DMSC will also check the integrity and the validity of the data and will make recommendations.

The trial steering committee consists of the clinical coordinators for each country (JG, PJ), the sponsor representative (CSB), and the trial statistician (AR).

All participants will be offered continuous follow-up after completion of the trial in the specialized lung transplant clinics in participating centers and are taken into account by patients in the vast majority. In Europe, lifelong center-based follow-up is the standard of care after lung transplantation to assure optimal patient management, quality management, and handling of post-trial complications. In both centers in case or irreversible graft loss, the possibility of re-do transplantation can be offered.

\section{Dissemination}

The results of the trial are planned to be presented at congresses and to be published in a medical journal. Furthermore, trial results are planned to be distributed via newsletter of the German Center of Lung Research. Guidelines of authorship of the Deutsche Forschungsgemeinschaft will be followed. Authorship will be determined according to the number of randomized centers between principal investigators. Trial data will be made 
available for publicity starting 1 year and ending 10 years after the publication of results upon request to the corresponding author.

There is no plan for using professional writing in the distribution of the results.

\section{Discussion}

This is the first prospectively randomized trial to investigate tailoring of immunosuppression by a DNA virus (torque teno virus) in comparison with conventional therapeutic drug monitoring in lung transplantation.

Viral infection may cause symptoms, but some viruses can be found in healthy individuals without causing disease. Torque teno virus (TTV) is a human DNA virus resulting in asymptomatic viremia. TTV viremia is frequently detected in the general population without associated symptoms or disease. Epstein-Barr virus (EBV) represents another DNA virus, has a high prevalence in adults, and causes latent infection in memory B cells after primary infection, and viremia is usually asymptomatic in the transplant population. The levels of EBV in the blood are correlated with the intensity of immunosuppression. Both TTV and EBV have therefore been suggested as surrogate markers of the net state of immunosuppression. In previous studies, TTV DNA in the blood but not EBV viremia is correlated positively with the intensity of immunosuppression after transplantation. Another argument against the use of EBV load as a surrogate marker of immunosuppression is the influence of valganciclovir on EBV load, a drug commonly used for preventing CMV infection after lung transplantation. Its use may reduce EBV load. In conclusion, TTV but not EBV DNA load better reflects the function of the immune system after lung transplantation. Immunosuppression depends on the type, combinations, and dosing of immunosuppressive treatment.

In lung transplant recipients, a large benefit may be expected by tailoring immunosuppression by DNA virus monitoring because immunosuppression is usually more intense in comparison with other forms of solid organ transplantation. This hypothesis is optimally being studied in a prospective manner in comparison with conventional therapeutic drug monitoring.

The guidance of immunosuppression by monitoring of TTV load will be chosen because in contrast to EBV, and cytomegalovirus (CMV) is expected to be measurable in the blood in the vast majority of lung transplant recipients and monitoring of this virus is therefore suitable for the guidance of immunosuppression. Since less than $25 \%$ of recipients are expected to develop CMV viremia during the first postoperative year, CMV load will not be suitable as a monitoring tool for immunosuppression. Furthermore, several publications have demonstrated changes in TTV load in relation to the intensity of immunosuppression [3, 7]. CMV load will be monitored routinely in addition to all recipients, and prophylaxis will be given. However, randomization will be stratified by high-risk CMV status (D+R-) (yes/no) as in the Vienna cohort [4] a lower TTV load was observed during CMV infections. Blinding of patients and/or staff will not be applied as the primary endpoint will be determined by using laboratory values in the CKD-EPI formula.

To compare the results regarding TTV levels between different transplantation centers, it is vital to reliably quantify the concentrations of TTV DNA in a standardized manner. In the VIGILung trial, this will be performed by central analysis of TTV load in a reference lab because real-time PCR is prone to inter-laboratory differences.

The effect size assumed for the sample size calculation was derived from the observed glomerular filtration rate (GFR) changes within 12 months in two recent immunosuppressive trials including reduced calcineurin inhibitor strategies $[8,13]$. All endpoints will be assessed by predefined definitions [9, 14-18]. The CKD-EPI method was chosen to estimate GFR because it is the most robust creatinine-based method and is established in clinical routine and trials in lung transplantation [19]. CLAD will be diagnosed by a persistent (at least 3 months) decline of FEV1 to $80 \%$ of baseline or below after adequate treatment of secondary causes such as infection, acute cellular/antibody-mediated rejection, or airway stenosis.

The authors recognize that the COVID-19 public health emergency may impact the conduct of the trial, e.g., by site personnel or trial participants become infected with COVID-19, quarantines, site closures, and travel limitations. Safety of trial participants is ensured by the prescription of immunosuppressants by local physicians and remote monitoring of patients by telephone and video consultation [20]. During the first wave of the pandemic, shortage in immunosuppressive medications was not noted in Germany and Austria. In-person visits by indications were still possible in both participating centers. Changes in the study visit schedules, missed visits, or patient discontinuations may lead to missing information. Temporary stopping of recruitment may limit the amount of missing data.

The results of this trial might have a large impact on therapeutic strategies for patients after lung transplantation. Furthermore, this study will contribute to improve evidence-based therapy in these patients. Study results are transferable to all other solid organ transplantations.

\section{Trial status}

The trial is recruiting patients since 28 July 2020 according to the VIGILung protocol version V03 from $05 \mathrm{No}$ vember 2019. Recruitment time is planned to be 39 months. 


\section{Supplementary Information}

The online version contains supplementary material available at https://doi. org/10.1186/s13063-020-04985-w.

Additional file 1. SPIRIT checklist. Filled out SPIRIT checklist for the VGILung trial protocol.

Additional file 2. Translation of the funding information

Additional file 3. Translation of the Ethics committee's vote of Hannover Medical School

Additional file 4. Translation of the Ethics committee's vote of Medical University of Vienna

Additional file 5. Tabular view from registration at clinicaltrials.gov concerning $\mathrm{WHO}$ trial dataset

\section{Abbreviations}

AE: Adverse event; AR: Adverse reaction; ATG: Antithymocyte globulin; BAL: Bronchoalveolar lavage; CA: Competent authority; CARV: Communityacquired respiratory viral infections; CKD-EPI: Chronic Kidney Disease Epidemiology Collaboration; CMV: Cytomegalovirus; CNI: Calcineurin inhibitor; CRF: Case report form; CsA: Ciclosporin A; DNA: Deoxyribonucleic acid; DSA: Donor-specific a; DSMC: Data Safety Monitoring Committee; DSUR: Development Safety Update Report; EBV: Epstein-Barr virus; EC: Ethics committee; EC-MPS: Enteric-coated mycophenolate sodium; FEV1: Forced expiratory volume in $1 \mathrm{~s}$; GCP: Good Clinical Practice; GFR: Glomerular filtration rate; HBsAG: Hepatitis B surface antigen; HCV: Hepatitis C virus; HHV6: Human herpesvirus 6; HIV: Human immunodeficiency virus; HLA: Human leukocyte antigen; ICH: International Conference on Harmonization; IS: Immunosuppression; ITT: Intention-to-treat; LC-MS: Liquid chromatography-mass spectrometry; LTx: Lung transplantation; MMF: Mycophenolate mofetil; MPA: Mycophenolic acid; $\mathrm{pO}_{2}$ : Oxygen partial pressure; PP: Per-protocol; SAE: Serious adverse event; $\mathrm{SaO}_{2}$ : Blood-oxygen saturation; SAR: Serious adverse reaction; SmPC: Summary of product characteristics; $\mathrm{SpO}_{2}$ : $\mathrm{SaO}_{2}$ measurement determined by pulse oximetry; SUSAR: Suspected unexpected serious adverse reaction; TAC: Tacrolimus; TBB: Transbronchial biopsy; TDM: Therapeutic drug monitoring; TLC: Total lung capacity; TTV: Torque teno virus; UAR: Unexpected adverse reaction; 6MWT: 6-minute walk test

\section{Acknowledgements}

None

Sponsor:

Philipps-University Marburg, Germany

Representative of the sponsor:

Koordinierungszentrum für Klinische Studien (KKS)

Philipps-Universität Marburg

Karl-von-Frisch-Str. 4

35043 Marburg, Germany

\section{Biological sampling}

Collection and storage of biological samples will be performed locally. In Hannover, sample collection, storage, and administration will be performed in the Hannover Unified Biobank (HUB, more information: https://www.mhh. de/institute-zentren-forschungseinrichtungen/hannover-unified-biobank-hub). All participants will give informed consent for the storage and processing of biological samples.

\section{Authors' contributions}

Conceptualization and primary investigator: JG. Methodology: JG, AR, KM, $\mathrm{KW}, \mathrm{CS}, \mathrm{SH}$, and PJ. Project administration: KW and SH. Writing-original draft: SH. Writing - review and editing: JG, AR, KM, KW, CS, SH, and PJ. The authors read and approved the final manuscript.

\section{Funding}

This study is funded by the Deutsche Forschungsgemeinschaft (DFG, German Research Foundation) - project-number 329015138.

The DFG have granted 1.006.701€ over a period of 36 months to our project as part of their "clinical trials" program. This funding will cover the costs for both scientific and medical personnel, including case payments for recruiting and treating individual patients.
Within the terms of the agreement, funding is subjected to bi-annual progress reporting. A prolongation of the application beyond the planned 36 months is permissible, subject to preliminary findings and agreed publication of these results. Open Access funding enabled and organized by Projekt DEAL.

\section{Availability of data and materials}

As the study is ongoing, data are not yet available. Materials about the study are available under the registration number: EudraCT-Number 2019-001770-29.

\section{Ethics approval and consent to participate}

The study will be conducted in accordance with the protocol, with the current version of the Declaration of Helsinki, ICH-GCP Guideline (International Conference on Harmonization - Good Clinical Practice), and applicable national laws and regulatory requirements.

The VIGILung study EudraCT No.: 2019-001770-29

Protocol version V03F, dated 05 November 2019 has been approved by the Ethics Committees of the Hannover Medical School, Germany (Nr. 8579_AMG_mono_2019) and the Medical University of Vienna (EK Nr.: 1127/ 2020) as well as by the German and Austrian Competent Authorities, Bundesinstitut für Arzneimittel und Medizinprodukte (Vorlage Nr. 4043539), and Bundesamt für Sicherheit im Gesundheitswesen BASG, reference 12760630 on 29 April 2020.

Any substantial amendments to the protocol will be submitted to the EC in accordance with national requirements. Additional study sites may only recruit patients if the sponsor already obtained approval for the site. Written informed consent will be obtained from all participants in the trial before inclusion. The patient information and consent form was approved by the Ethics Committee of the Hannover Medical School and Medical University of Vienna. All patients after lung transplantation are followed in specialized follow-up clinics in participating centers. During the first visit after discharge from the hospital which is as early as 21 days after the surgical procedure, patients will be approached about the trial by transplant pulmonologists who are investigators of the trial. Informed consent of participants will also be obtained by transplant pulmonologists who are also investigators of the trial. Screening of patients will be discussed before the visit during team meetings. Patients have already been informed by newsletters, flyers in the waiting area, and social media. It is estimated to screen 250 patients to include 144 patients from screening procedures from previous trials. Any ancillary planned studies using patient data will be included in further informed consent forms of the trial. At this point, no ancillary studies are planned so far.

\section{Consent for publication}

The ethical approval and patient information include consent to publish the collected data.

\section{Competing interests}

The authors declare to have no competing interests.

\section{Author details}

'Department of Respiratory Medicine OE6870, Hannover Medical School, 30625 Hannover, Germany. ${ }^{2}$ BREATH (Biomedical Research in End-stage and obstructive Lung Disease Hannover), Hannover, Germany. ${ }^{3}$ Coordinating Centre for Clinical Trials Marburg (KKS Marburg), Philipps-University Marburg, Marburg, Germany. ${ }^{4}$ University of Giessen and Marburg Lung Center (UGMLC), University Hospital Giessen, Justus Liebig University of Giessen, Giessen, Germany. ${ }^{5}$ Division of Thoracic Surgery, Medical University of Vienna, Vienna, Austria.

Received: 20 August 2020 Accepted: 18 December 2020

Published online: 11 January 2021

\section{References}

1. Ivulich S, Westall G, Dooley M, Snell G. The evolution of lung transplant immunosuppression. Drugs. 2018;78(10):965-82.

2. Yusen RD, Christie JD, Edwards LB, Kucheryavaya AY, Benden C, Dipchand $\mathrm{Al}$, et al. The Registry of the International Society for Heart and Lung Transplantation: Thirtieth Adult Lung and Heart-Lung Transplant Report-2013; focus theme: age. J Heart Lung Transplant. 2013;32(10):965-78. 
3. Gorzer I, Jaksch P, Kundi M, Seitz T, Klepetko W, Puchhammer-Stockl E. Pretransplant plasma torque teno virus load and increase dynamics after lung transplantation. PLoS One. 2015;10(3):e0122975.

4. Gorzer I, Jaksch P, Strassl R, Klepetko W, Puchhammer-Stockl E. Association between plasma torque teno virus level and chronic lung allograft dysfunction after lung transplantation. J Heart Lung Transplant. 2017;36(3): 366-8.

5. Jaksch P, Kundi M, Gorzer I, Murakozy G, Lambers C, Benazzo A, et al. Torque teno virus as a novel biomarker targeting the efficacy of immunosuppression after lung transplantation. J Infect Dis. 2018;218(12): $1922-8$.

6. Ravaioli M, Neri F, Lazzarotto T, Bertuzzo VR, Di Gioia P, Stacchini G, et al. Immunosuppression modifications based on an immune response assay: results of a randomized, controlled trial. Transplantation. 2015;99(8):1625-32.

7. De Vlaminck I, Khush KK, Strehl C, Kohli B, Luikart H, Neff NF, et al. Temporal response of the human virome to immunosuppression and antiviral therapy. Cell. 2013;155(5):1178-87.

8. Gottlieb J, Neurohr C, Muller-Quernheim J, Wirtz H, Sill B, Wilkens H, et al. A randomized trial of everolimus-based quadruple therapy vs standard triple therapy early after lung transplantation. Am J Transplant. 2019;19(6):175969.

9. Levey AS, Stevens LA, Schmid CH, Zhang YL, Castro AF 3rd, Feldman HI, et al. A new equation to estimate glomerular filtration rate. Ann Intern Med. 2009;150(9):604-12.

10. Jaksch P, Ankersmit J, Scheed A, Kocher A, Murakozy G, Klepetko W, et al. Alemtuzumab in lung transplantation: an open-label, randomized, prospective single center study. Am J Transplant Off J Am Soc Transplant Am Soc Transplant Surg. 2014;14(8):1839-45.

11. Hachem RR, Yusen RD, Chakinala MM, Meyers BF, Lynch JP, Aloush AA, et al. A randomized controlled trial of tacrolimus versus cyclosporine after lung transplantation. J Heart Lung Transplant. 2007;26(10):1012-8.

12. Maggi F, Pifferi M, Fornai C, Andreoli E, Tempestini E, Vatteroni $M$, et al. TT virus in the nasal secretions of children with acute respiratory diseases: relations to viremia and disease severity. J Virol. 2003;77(4):2418-25.

13. Strueber M, Warnecke G, Fuge J, Simon AR, Zhang R, Welte T, et al. Everolimus versus mycophenolate mofetil de novo after lung transplantation: a prospective, randomized, open-label trial. Am J Transplant. 2016;16(11):3171-80

14. Holland AE, Spruit MA, Troosters T, Puhan MA, Pepin V, Saey D, et al. An official European Respiratory Society/American Thoracic Society technical standard: field walking tests in chronic respiratory disease. Eur Respir J. 2014; 44(6):1428-46.

15. Humar A, Michaels M, Monitoring AIWGolD. American Society of Transplantation recommendations for screening, monitoring and reporting of infectious complications in immunosuppression trials in recipients of organ transplantation. Am J Transplant. 2006;6(2):262-74.

16. Ljungman P, Boeckh M, Hirsch HH, Josephson F, Lundgren J, Nichols G, et al. Definitions of cytomegalovirus infection and disease in transplant patients for use in clinical trials. Clin Infect Dis. 2017;64(1):87-91.

17. Verleden GM, Glanville AR, Lease ED, Fisher AJ, Calabrese F, Corris PA, et al. Chronic lung allograft dysfunction: definition, diagnostic criteria, and approaches to treatment-a consensus report from the pulmonary council of the ISHLT. J Heart Lung Transplant. 2019;38(5):493-503.

18. Stewart S, Fishbein MC, Snell Gl, Berry GJ, Boehler A, Burke MM, et al. Revision of the 1996 working formulation for the standardization of nomenclature in the diagnosis of lung rejection. J Heart Lung Transplant. 2007;26(12):1229-42

19. Degen DA, Janardan J, Barraclough KA, Schneider HG, Barber T, Barton H, et al. Predictive performance of different kidney function estimation equations in lung transplant patients. Clin Biochem. 2017;50(7-8):385-93.

20. Kayser M, Valtin C, Greer M, Karow B, Fuge J, Gottlieb J. Video consultation during the COVID-19 pandemic: a single center's experience with lung transplant recipients. Telemed J E Health. 2020. https://doi.org/10.1089/tmj. 2020.0170 Online ahead of print.

\section{Publisher's Note}

Springer Nature remains neutral with regard to jurisdictional claims in published maps and institutional affiliations.

\section{Ready to submit your research? Choose BMC and benefit from}

- fast, convenient online submission

- thorough peer review by experienced researchers in your field

- rapid publication on acceptance

- support for research data, including large and complex data types

- gold Open Access which fosters wider collaboration and increased citations

- maximum visibility for your research: over $100 \mathrm{M}$ website views per year

At $\mathrm{BMC}$, research is always in progress.

Learn more biomedcentral.com/submissions 